\title{
A Design of HD Video Compression and Processing System Based on TMS320DM6467 Processor
}

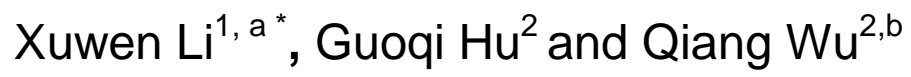 \\ ${ }^{1}$ College of Life Science and Bioengineering, Beijing University of Technology, China \\ ${ }^{2}$ College of Electronic Information and Control Engineering, Beijing University of Technology, China \\ alixuwen@bjut.edu.cn, bwuqiang@bjut.edu.cn
}

Keywords: HD, video compression, H.264, BT.1120

Abstract. This paper proposed a design of HD video compression and processing system due to the process of large amount of data, high flexibility problem during video compression and video processing. This design apply TMS320DM6467 dual-core DaVinci processor. After the research of VPIF interface and video compression, this paper proposed a design which can meet the system requirements.

\section{Introduction}

With the development of multimedia technology, the requirements of various fields for the video data acquisition, processing and transmission continues to improve, especially in the field of aerospace [1] [2]. Application of aerospace requirements more stable, flexible, complex system. The TI Company produced several high performance digital signal processors based on DaVinci Technology [3], which were used in many domestic product. Because the DM6467 processor has a wealth of experience in dealing with anti-radiation in the outer space and this processor use ARM core and DSP core architecture [4] which combines the flexible control unit and high performance signal processing unit, this promotes the multi-media development [5]. This paper research the HD video compression and processing system based on TMS320DM6467. This paper designed two different video processing schemes according to the demand of the system.

\section{System Framework}

This system process image acquired by CMOS sensor according to the system platform and transfer the data to system platform as shown in Figure 1. This paper proposed the solution to the way of video compression and processing and designed two sets of video processing scheme according to the two different modes of the system requirements.

The video data flow is shown in Figure 2. The CMOS sensor transfer the image data to the signal processing board and encode with YCbCr to YUV and BT.1120 in the signal processing board. Then transfer the video data to the video compression board through the VPIF interface. The video compression board processes the video data according to the instruction, including YUV format conversion, H.264 encoding, image algorithm, transferring the video data to the system platform.

\section{The Design of Video Capturing and Encoding}

\section{A. The design of video capture}

The system use HD CMOS its output formats meet YCbCr4:2:2. The Y and C component data quantization are both 8 bit. The data synchronization clock is $80 \mathrm{MHz}$. this paper have a good design of this non-standard video data real-time transmission system in this section.

The video compression board use one TMS320DM6467 DaVinci processor which has a VPIF interface exclusively for video data transmission as shown in Figure 3. The VPIF interface supports SDTV, HDTV data input/output and RAW capture mode. 


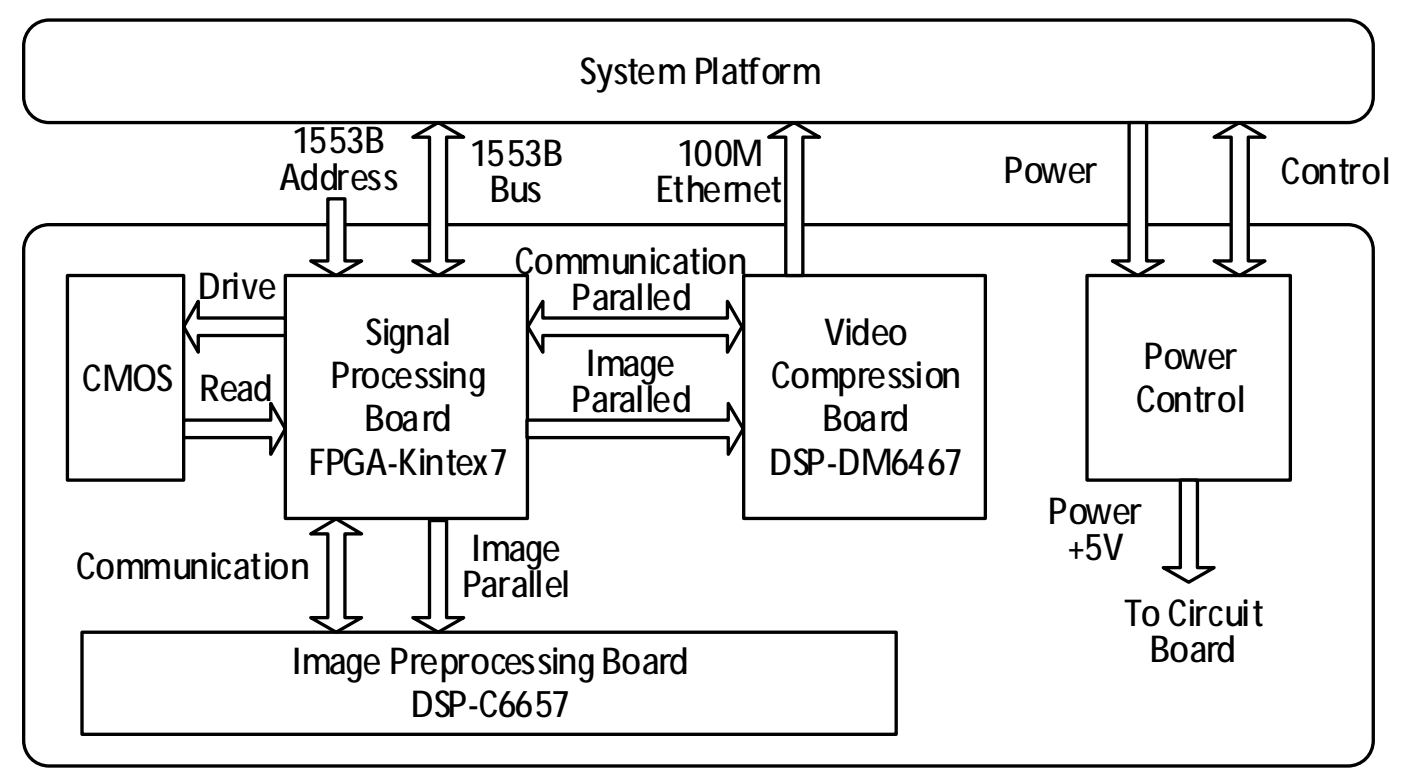

Fig. 1 System Framework

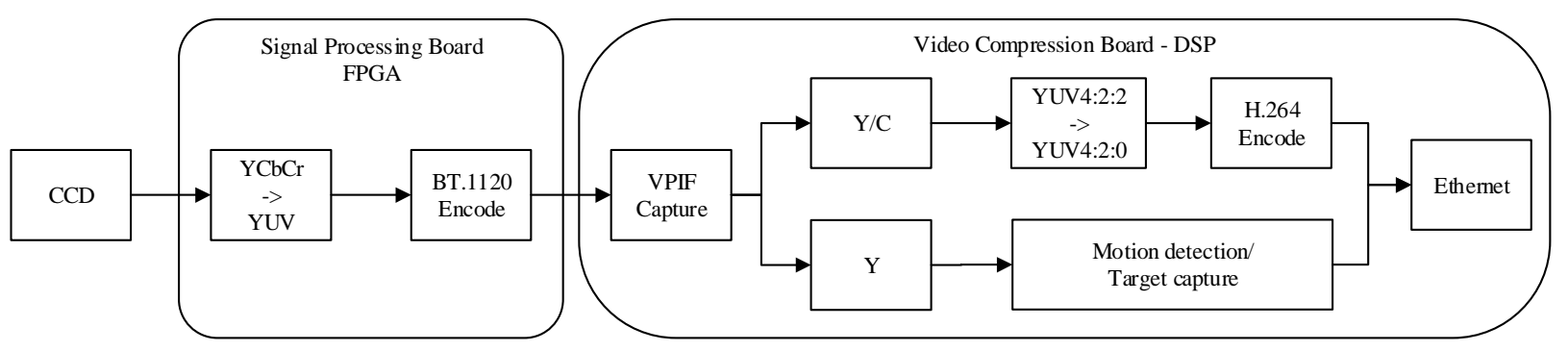

Fig. 2 Data Flow

Because the image resolution of the system is 1920x1080, this system adopts HDTV mode. In this mode the transmission of $\mathrm{Y}$ component data and $\mathrm{C}$ component data is separated $\mathrm{Y}$ component data is transmitted through VPIF channel 0, the $\mathrm{C}$ component data is transmitted through VPIF channel 1.

(1) SDTV 1ch input and output

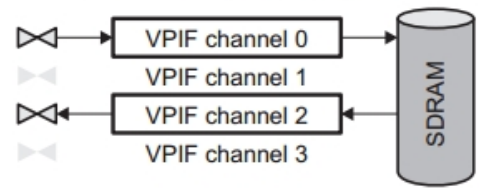

(2) SDTV 2ch input and output

(3) HDTV 1ch input and output
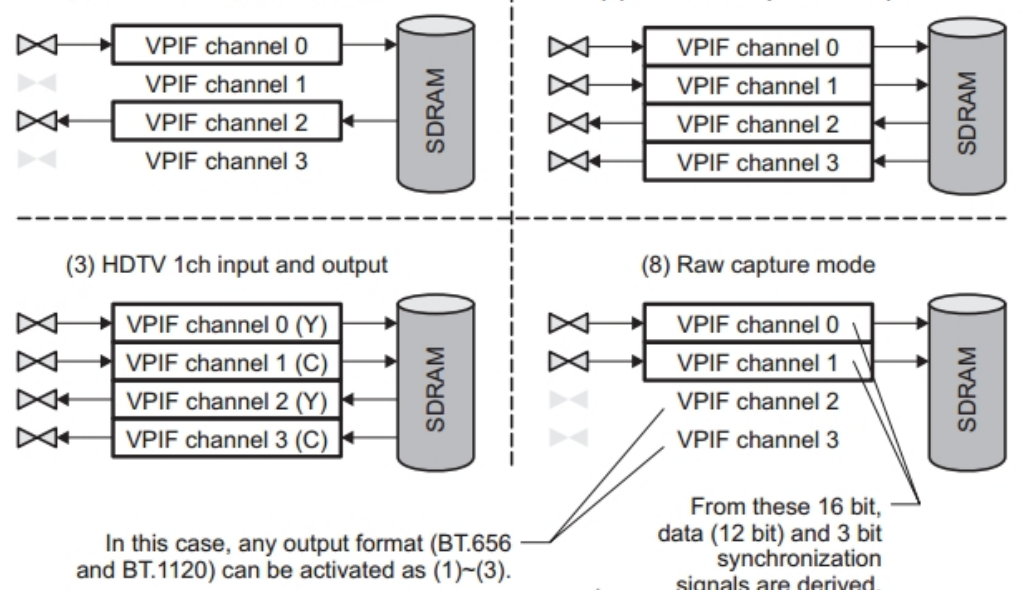

(8) Raw capture mode

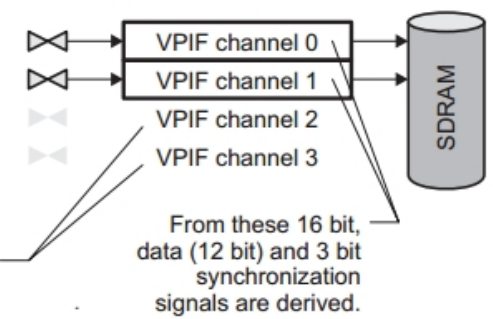

Fig. 3 Input and Output Channels of VPIF

Using the VPIF port has to consider the problem of 27MHz system clock, using ether SDTV input model or HDTV input model as shown in Figure 4. As mentioned in official document, a stable $27 \mathrm{MHz}$ system clock was essential due to the real-time transmission of the encoded data. So we use the $27 \mathrm{MHz}$ clock source as system clock input. 
In the HDTV input mode, the input video format has to be of BT.1120 and the Video Input clock signal uses $74.25 \mathrm{MHz}$ or $148.5 \mathrm{MHz}$, but the DSP chip only supports up to $99 \mathrm{MHz}$ of the VPIF. So this paper has to adopt $74.25 \mathrm{MHz}$ video synchronization clock. In the International Telecommunication Union HDTV related RECOMMENDATION ITU-R BT.1120-8, the standard video progressive scan frame rate supports $60 \mathrm{fps}, 50 \mathrm{fps}$, 30fps, $25 \mathrm{fps}$, 24fps, synchronous clock supports $74.25 \mathrm{MHz}$ or $148.25 \mathrm{MHz}$. But the frame rate transmission of this system platform needs to switch among 5fps, 10fps, 20fps, and the synchronous clock needs to be adjusted according to the FPGA part.

In summary, the difficulties of video transmission system lies in the design of synchronous clock rate and video transmission standards does not match, video transmission and standards does not match.

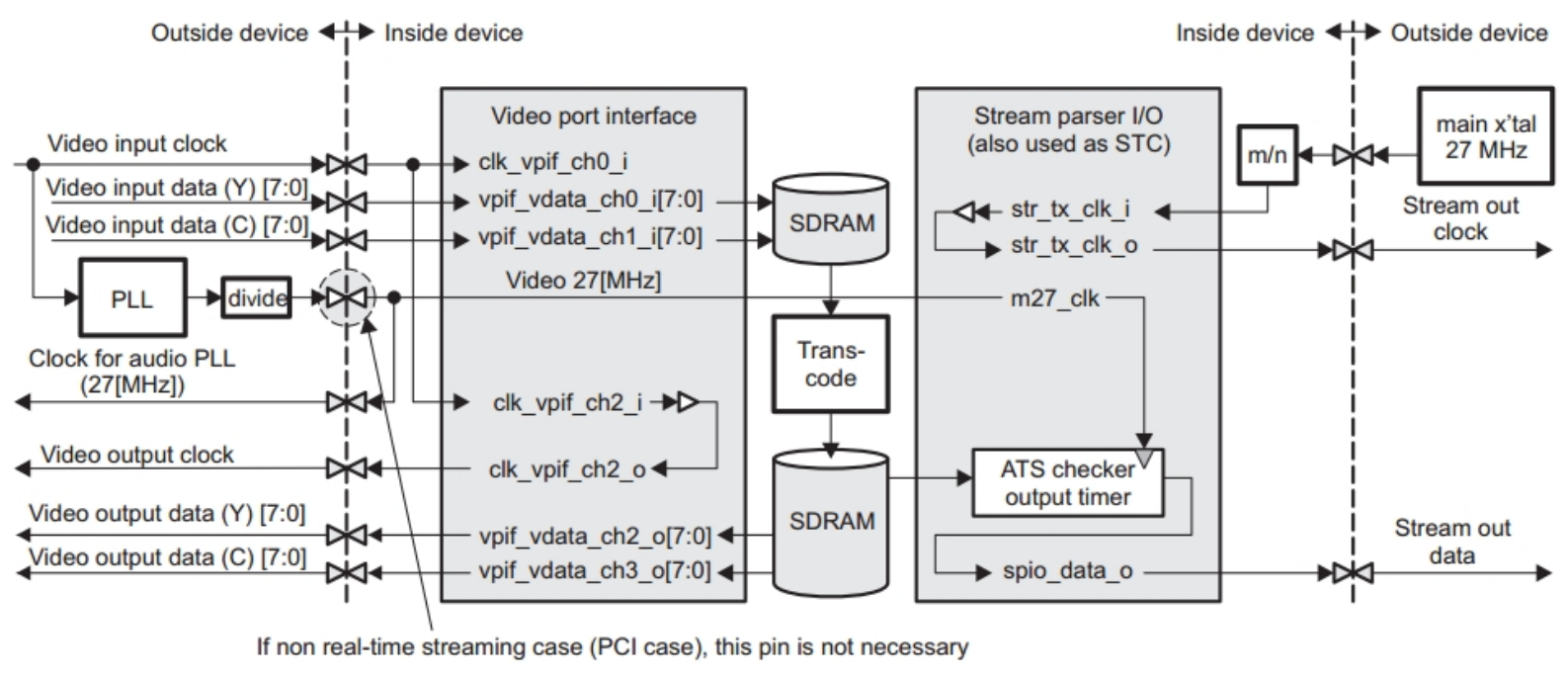

Fig. 4 Clock Control on Video Input and Output with HDTV Encoding

\section{B. BT.1120 Encoding}

Because of the synchronous clock of CMOS video data output is $80 \mathrm{MHz}$, the signal processing board has no enough storage space to store the video data locally and send out in the format of BT.1120 format. So the $80 \mathrm{MHz}$ video synchronization clock is used to send data. In addition, the format of BT.1120 contain the synchronizing information, the receiver identify the video data by the embedded EAV、SAV synchronous signals. So there is no strictly requirement for video frame. According to the ITU-R BT.1120-8 and TI TMS320DM6467 Digital Media System-on-Chip data, the implement in the FPGA BT.1120 video encoding sequence table is shown in Table 1.

H.264 encoding by two Mealy status machines based on the FPGA. One Mealy status machines used for controlling the switch among the different line of the same image. The other one Mealy status machines used for controlling the switch among the different regions of the same line.

According to BT.1120 data format, the data of the line is divided into five regions, namely, EAV data area, the auxiliary data region, SAV data area, an active data area, and IDLE, using a counter for counting a column value of the image, and according to the counter values to achieve the jump between the states. The timing logic of FPGA is programed according to Table 1.

\section{DSP Video Compression and Processing}

Video data in video compression board is processed as shown in Figure 2. One is compressing the $\mathrm{Y}$ and $\mathrm{C}$ component in $\mathrm{H} .264$ and is output through Ethernet; the other is processing the $\mathrm{Y}$ component using image algorithm and the results and Y component is output through Ethernet. The two mode is selected based on the instruction system platform, the same time only has one mode.

When the H.264 compression is essential, VPIF interface receives the Y component and C component. The size of one frame takes $1920 \mathrm{X} 1080 \mathrm{X} 2$ bytes. The first $1920 \mathrm{X} 1080$ bytes are Y 
component. The second 1920X 1080 bytes are $\mathrm{C}$ component. The format of the video data received by VPIF are YUV4:2:2.Then the data format is converted to YUV4:2:0 and encoded of H.264 using the V4L2 library. Finally the encoded data are send to the system platform through the Ethernet as shown in Figure 5.

Table 1 BT.1120 Timing

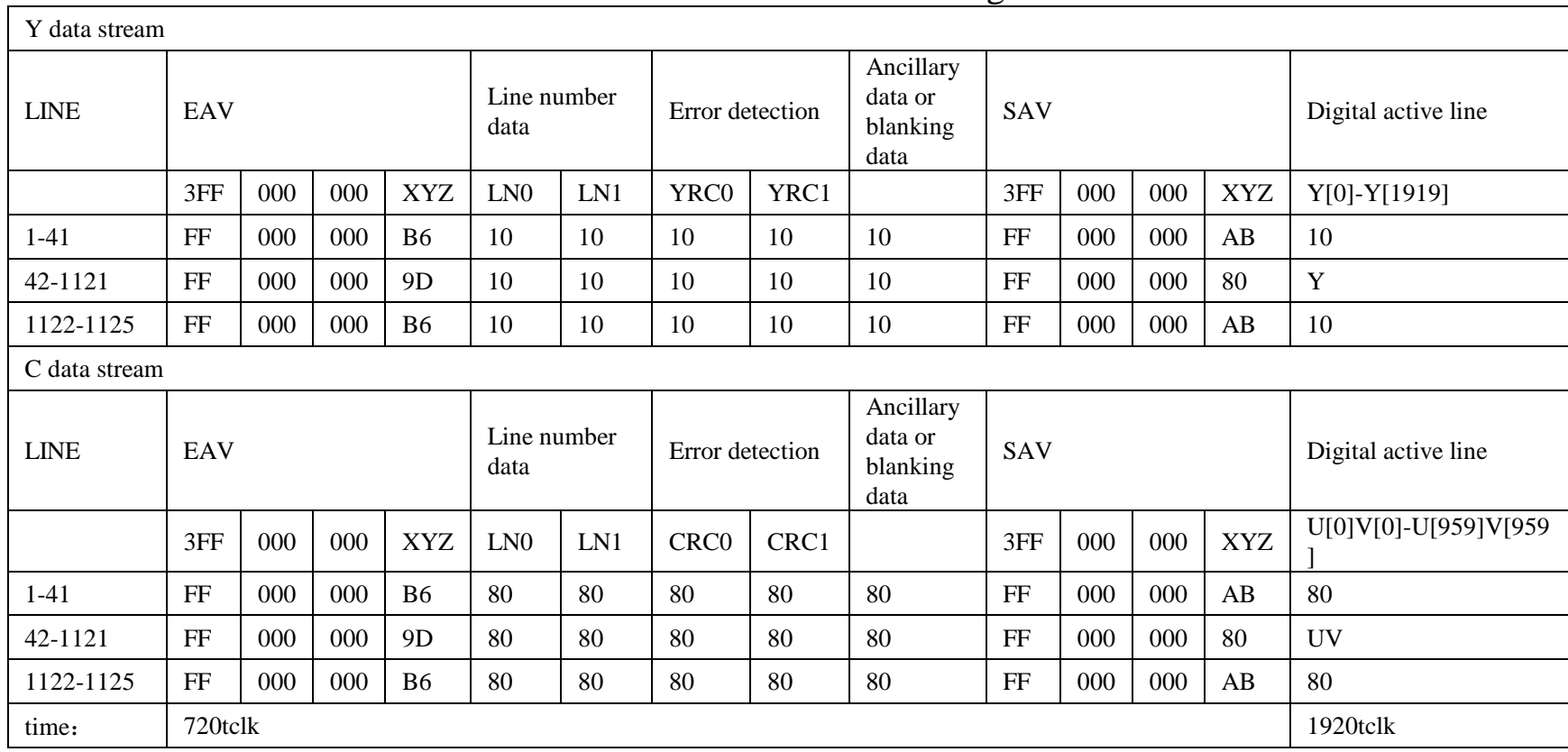

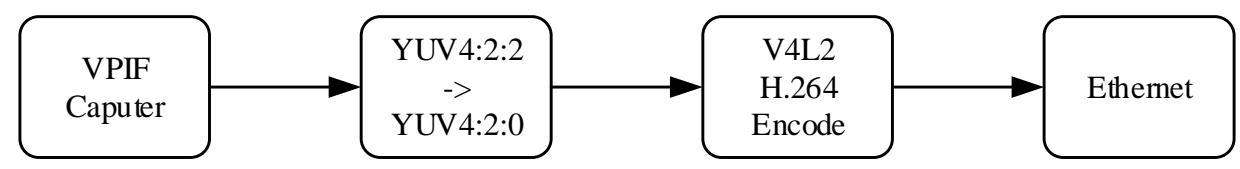

Fig. 5 Framework of H.264 Video Compression

When the Y component is processed using image algorithm, VPIF interface receives the Y component. The size of one frame is $1920 \mathrm{X} 1080$ bytes. The moving detection, target capturing algorithm are using on this image and the result are send to the system platform through Ethernet.

\section{System Experiment}

The video compression board is shown in Figure 6. This board will send the video data through Ethernet to the system platform, and send the system debugging information through the serial port to the system platform. As shown in Figure 7, the system platform received the debugging information. The Ethernet transmission rate is less than $10 \mathrm{Mbps}$ and the video frame rate can also the normal switching. The video decoding software on the system platform is as shown in Figure 8. The software is decoding the HD video which send by the video compression board.

After extensive testing, the system can make the correct data processing in different Telemetry Command and the results can be seen from the video decoding software in system platform.

\section{Summary}

This paper design the non-standard video data transmission method based on TMS320DM6467 VPIF interface. A large amount of experiments has proved that this system is stable in it application. According to the system design requirements, two kinds of video data modes are achieved. The whole system has passed the test. The design fully realize all the needs. The parameters are also qualified. 


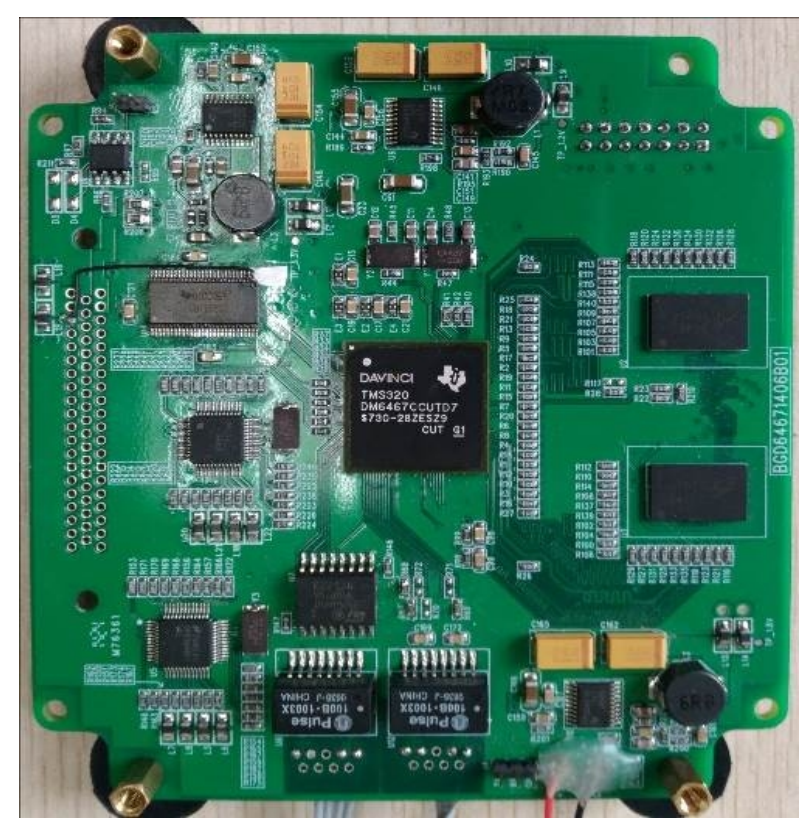

Fig. 6 Video Compression Board

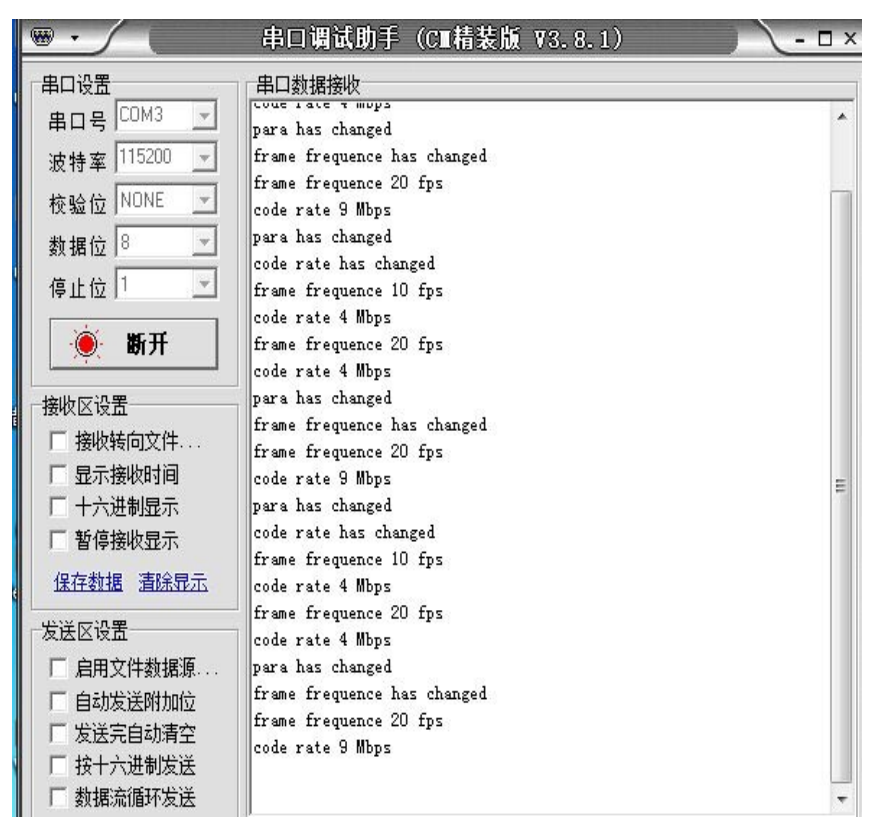

Fig. 7 System Debug Information

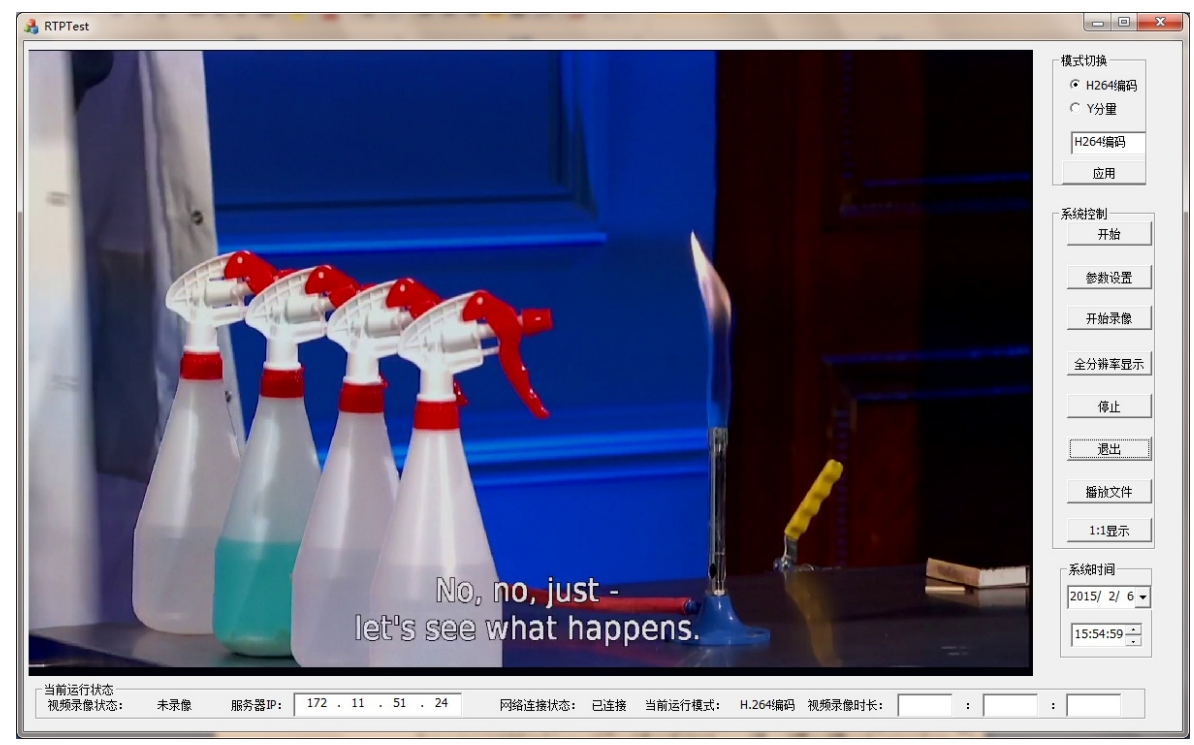

Fig. 8 Video Decoding and Playback Software

\section{References}

[1] Yao Chunlian, Guo Keyou, Jiang Huming, Design of embedded electronic video processing system based on DM6467. Lecture Notes in Electrical Engineering, v178 LNEE, VOL. 3, p487-492, 2013.

[2] Liu Hui, Zeng Hang-Cheng, Pu Bu, Implementation and optimization of H.264 encoder based on TMS320DM6467. Lecture Notes in Electrical Engineering, v136 LNEE, p465-472, 2012.

[3] Wang Jian, Hua Gang, Implementing high definition video codec on TI DM6467 SOC, 2008 IEEE International SOC Conference, SOCC, p193-196, 2008.

[4] Chen Xiao-Lin, Zhang Shan-Cong, Liu Jie, Design of UAV video compression system based on H.264 encoding algorithm, Proceedings of 2011 International Conference on Electronic and Mechanical Engineering and Information Technology, EMEIT 2011, v5, p2619-2622, 2011.

[5] RECOMMENDATION ITU-R BT.1120-8, Digital interfaces for HDTV studio signals, 2012. 